\section{Fotoféresis en el tratamiento de Síndrome de Sézary. Caso clínico}

\author{
MONTSERRAT MOLGÓ ${ }^{1}$, ALEJANDRA JAQUE $^{1}$, \\ VERÓNICA VIAL ${ }^{2}$, MAURICIO OCQUETEAU ${ }^{3}$, JAIME PEREIRA ${ }^{3}$, \\ MAYLING CHANG ${ }^{3}$, SERGIO GONZÁLEZ ${ }^{4}$
}

\section{Sézary syndrome treated with extracorporeal photopheresis. Report of one case}

Sézary syndrome (SS) is an unusually aggressive T-cell lymphoma characterized by the triad of erythroderma, the presence of more than 1,000 Sézary cells in peripheral blood and lymphadenopathies. It is accompanied by generalized pruritus and poor quality of life. The management of SS depends on its stage, patient comorbidities, and treatment availability. Extracorporeal photopheresis $(E C P)$ is the first line of treatment for patients with T-cell lymphomas in stage IVA1, IVA2 or SS. This treatment comprises three phases: leukapheresis, photoactivation and subsequent reinfusion of lymphocytes. As it is an immunomodulatory therapy it does not produce generalized immunosuppression. We report a 76 year-old male with SS stage IIIb initially treated with 12 sessions of ultraviolet phototherapy without response. After 10 well-tolerated sessions of $E C P$, itching and skin lesions eventually disappeared.

(Rev Med Chile 2015; 143: 1449-1458)

Key words: Lyphoma, T cell; Sézary Syndrome; Photopheresis.
'Departamento de Dermatología, Facultad de Medicina, Pontificia Universidad Católica de Chile. ¿Universidad de los Andes, Chile. ${ }^{3}$ Departamento de Hematología y Oncología, Facultad de Medicina, Pontificia Universidad Católica de Chile.

${ }^{4}$ Departamento de Anatomía Patológica, Facultad de Medicina, Pontificia Universidad Católica de Chile.

Los autores no refieren conflicto de interés.

Recibido el 6 de enero de 2015, aceptado el 15 de julio de 2015.

Correspondencia a: Dra. Montserrat Molgó Novell Teléfono: 56223548492 montsemolgo1@gmail.com
$\mathrm{E}$ 1 síndrome de Sézary (SS) es un subtipo agresivo, poco frecuente, de linfoma cutáneo primario de células T (LCCT) diferente a la micosis fungoide (MF). Se presenta clínicamente como una eritrodermia exfoliativa, asociada a clones de células T (células de Sézary) circulando en el torrente sanguíneo, además de linfadenopatías.

A continuación se presenta un caso de SS tratado con fotoféresis en nuestro centro.

\section{Caso clínico}

Paciente de 76 años de edad, con antecedentes de EPOC y enfermedad coronaria. A principios de enero del año 2013 comenzó con cuadro caracterizado por prurito, sin lesiones cutáneas, algunos episodios de síndrome febril, fatiga y disminución de apetito, lo que originó múltiples consultas. En mayo del mismo año, se agregó compromiso cutáneo progresivo que finalizó en eritrodermia. Al examen físico, destacaba xerosis marcada, signos de grataje, eritema generalizado intenso y adenopatías cervicales e inguinales palpables. En junio de 2013 fue realizada una biopsia de piel, cuyo informe inicial fue interpretado como una reacción adversa a medicamentos; sin embargo, el hemograma mostró 30\% de linfocitos patológicos por lo que al reevaluar la biopsia se informaron linfocitos grandes, hipercromáticos, atípicos, tipo células de Sézary (Figura 1). La citometría de flujo (CMF) en sangre periférica mostró $11 \%$ de células de Sézary.

Se solicitó tomografía de cuello, tórax, abdomen y pelvis, en la cual destacaban pequeñas adenopatías en ambas cadenas yugulares, triángulos posteriores del cuello, supraclaviculares e inguinales en contexto de un síndrome linfoproliferativo. La biopsia de médula ósea fue negativa para linfoma; serología para HTLV-1, virus Epstein-Barr y citomegalovirus fueron negativas.

Se diagnosticó SS etapa IIIb $\left(\mathrm{T} 4-\mathrm{N}_{1}-\mathrm{M}_{0}-\mathrm{B}_{1}\right)$, ingresó al sistema GES (garantías explícitas en 
salud) en julio de 2013 e inició tratamiento con PUVA (12 sesiones) asociado a quimioterapia con clorambucil y prednisona. Debido a la mala respuesta, fue derivado a nuestro centro para evaluación y manejo. $\mathrm{Al}$ ingreso, el paciente presentaba eritrodermia, signos de grataje, xerosis

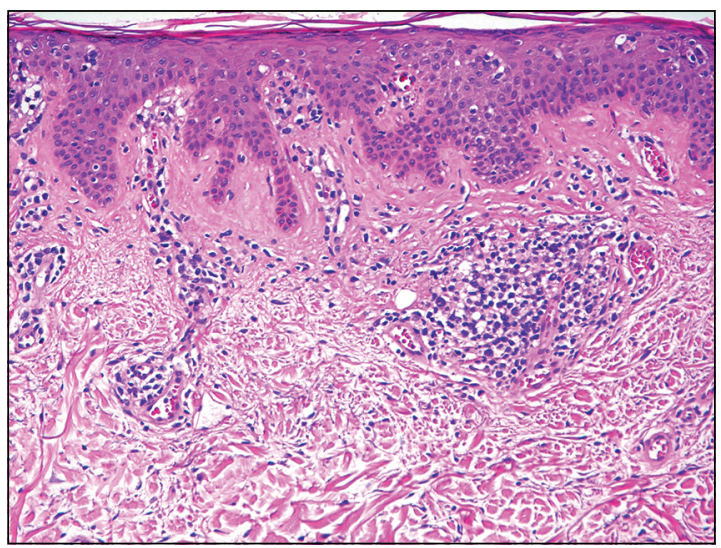

Figura 1. Histología pre- tratamiento: Infiltración por linfocitos atípicos, perivasculares, con fibrosis papilar y leve epidermotropismo. HE, 200x.

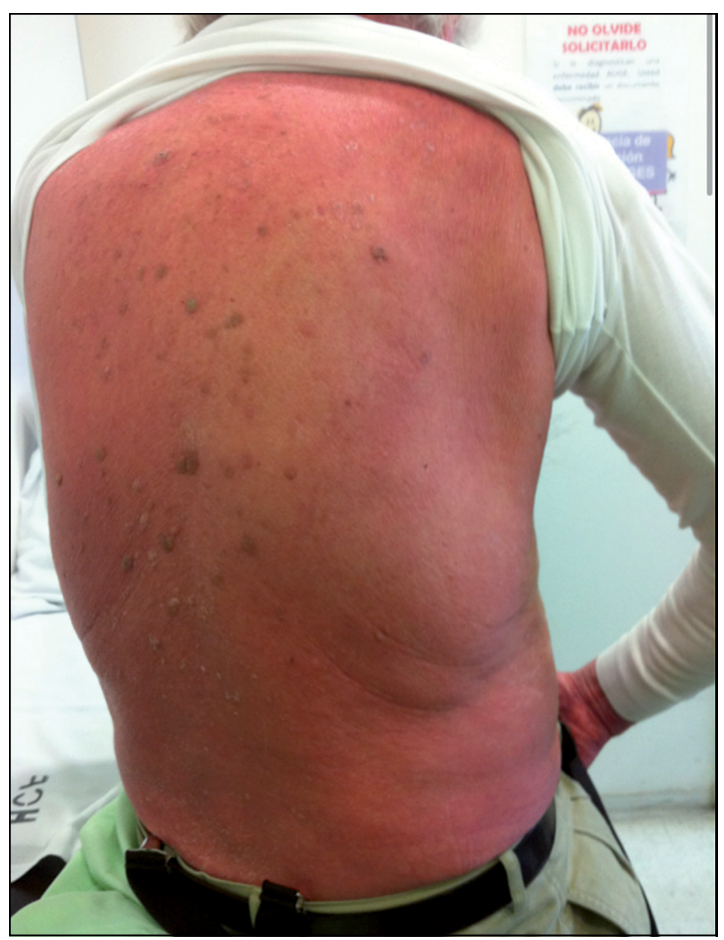

Figura 2. Octubre 2013: eritrodermia, xerosis marcada y placas hiperqueratósicas en zona dorso lumbar. y adenopatías (Figura 2). Además, presentaba un nódulo crateiforme de $7 \mathrm{~mm}$ de diámetro en ala nasal derecha, clínicamente sospechoso de un carcinoma espinocelular y traía un nuevo hemograma con 50\% de linfocitos patológicos y una LDH de 296 U/L. En conjunto con el equipo de Hemato-Oncología, se decidió, en octubre de 2013, iniciar terapia de fotoféresis extracorpórea (FEC) con una frecuencia de 2 sesiones cada 15 días, completando 10 sesiones en 2 meses y medio. En cada sesión se realizó una unipunción periférica, se extrajo un volumen aproximado de tratamiento de $260 \mathrm{ml}$, se utilizó $4,4 \mathrm{ml}$ de methoxalen y heparina como anticoagulante, con una duración promedio de de $3 \mathrm{~h}$. Se asoció manejo del prurito con anti H1 (hidroxicina) y emolientes tópicos. El paciente presentó buena respuesta, logrando controlar en $100 \%$ el prurito, con regresión completa de las lesiones cutáneas (Figuras 3 y 4). Se realizó una nueva biopsia post tratamiento que mostró atrofia epidérmica con fibrosis laxa superficial y fibroblastos sin linfocitos atípicos (Figura 5). La CMF mostró disminución de las células de Sézary de $11 \%$ inicial a $0,7 \%$ al terminar las sesiones de FEC (Tabla 1). Los linfocitos patológicos en hemograma disminuyeron de $50 \%$ a $0 \%$ en 4 meses (Tabla 1 ). En marzo

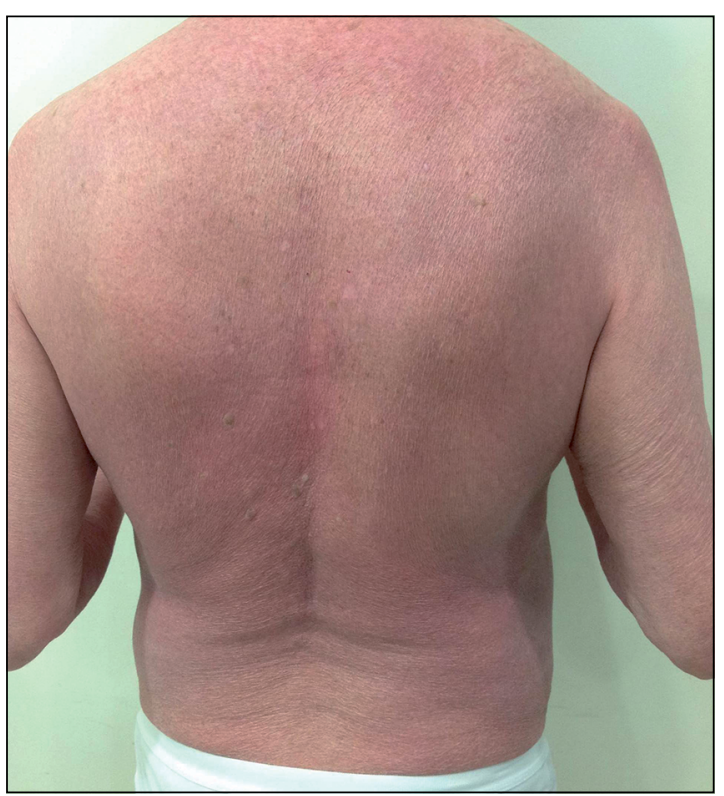

Figura 3. Diciembre 2013: Disminución del eritema y de las placas hiperqueratósicas en región dorso lumbar. 
Tabla 1. Resumen de evolución de síntomas y exámenes

\begin{tabular}{|lccccc|}
\hline Fecha & Xerosis & Eritema & Prurito & Hemograma & CMF \\
\hline Octubre de 2013 (previo inicio FEC) & +++ & +++ & $10 / 10$ & $50 \%$ & $11 \%$ \\
Diciembre de 2013 & ++ & ++ & $6 / 10$ & $25 \%$ & - \\
Enero de 2014 (terminada la FEC) & + & + & $1 / 10$ & $0 \%$ & $0,70 \%$ \\
Octubre de 2014 & - & - & $0 / 10$ & $0 \%$ & $0 \%$ \\
\hline
\end{tabular}

Hemograma: indica el \% de células de Sézary. CMF: Citometría de flujo; Indica el porcentaje en sangre periférica de células linfoides de estirpe T que presentan inmunofenotipo compatible con células de Sézary.

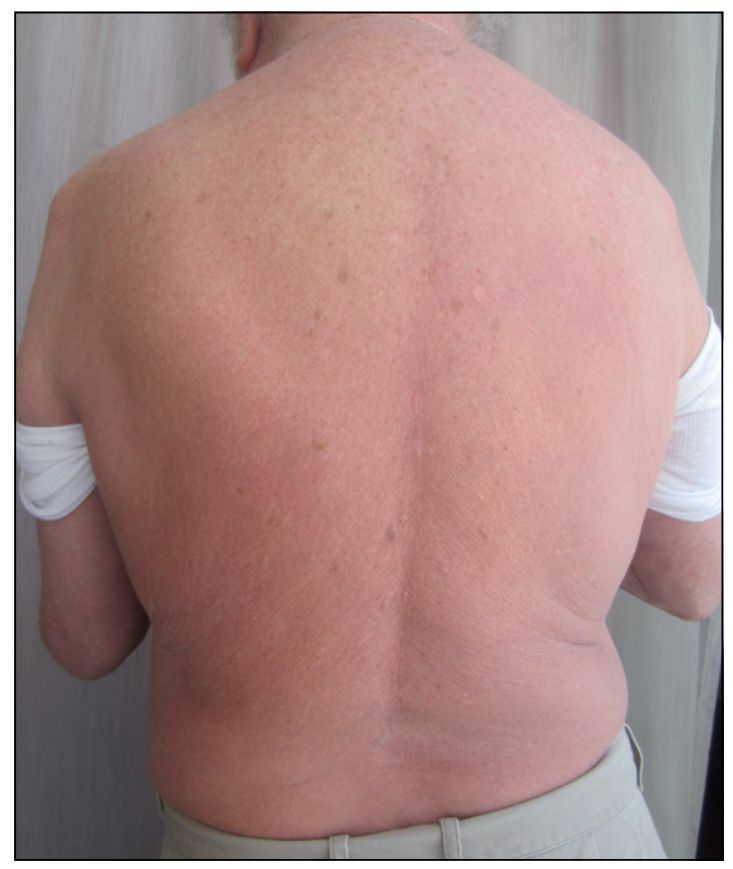

Figura 4. Enero 2014: Sin lesiones cutáneas, escasa xerosis.

de 2014, se realizó biopsia de lesión de ala nasal derecha que mostró un carcinoma espinocelular, tratado con haz de electrones por 6 semanas con excelente respuesta. Actualmente, lleva 11 meses de remisión (Figura 6). Última CMF de octubre de 2014 no mostró células de Sézary. Persiste en tratamiento con clorambucil y prednisona.

\section{Discusión}

En todo paciente que presente eritrodermia debe considerarse el diagnóstico de SS. La evaluación inicial necesariamente incluye una biopsia con evaluación de la morfología, inmunofenotipo

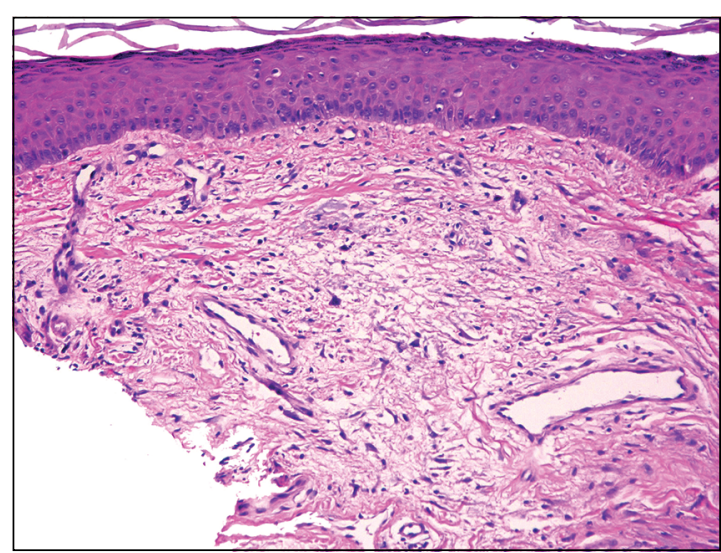

Figura 5. Histología post- tratamiento: Atrofia epidérmica con fibrosis laxa superficial y fibrobastos; no se observan linfocitos atípicos. HE, 200x.

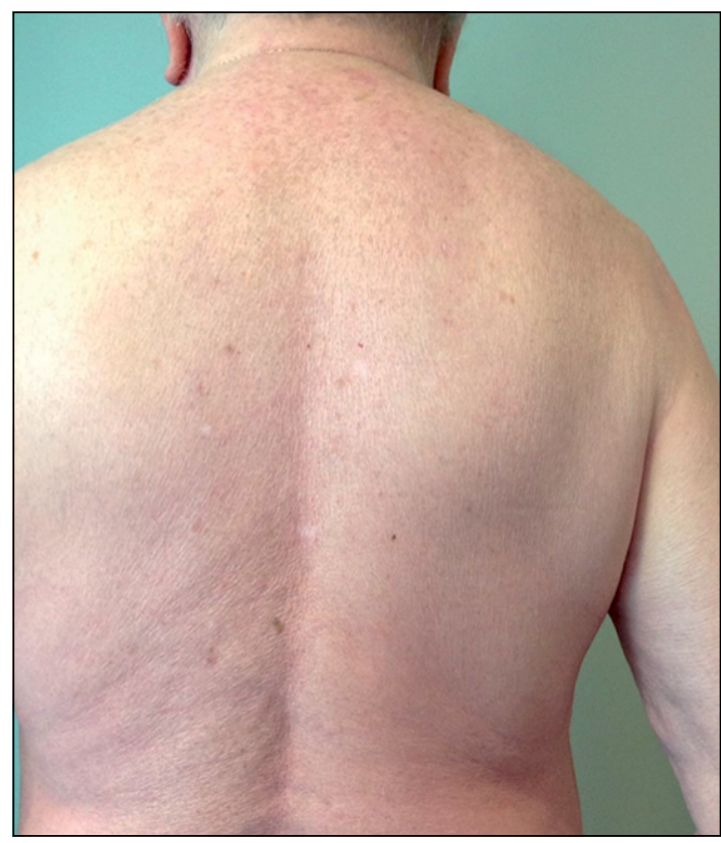

Figura 6. Octubre 2014: Sin lesiones cutáneas. 
y clonalidad de los infiltrados celulares. Si los resultados cutáneos son sugerentes de un linfoma cutáneo de células $\mathrm{T}$ o MF, debe realizarse un estudio citológico, citometría de flujo y clonalidad de sangre periférica para confirmar el diagnóstico (el reordenamiento clonal en sangre debiera ser el mismo que el cutáneo). Además, en pacientes con biopsia compatible con MF eritrodérmica, o eritrodermia asociada a adenopatías, se recomienda el estudio de imágenes, con una tomografía computarizada, una emisión tomografica combinada de positrones (PET) o una combinación de ellas ${ }^{1}$.

El consenso de la ISCL/EORTC publicado el año $2007^{1}$ determina como criterio diagnóstico de SS la presencia de:

- Eritrodermia.
- Reordenamiento clonal de receptor de células T en la sangre identificado por PCR o Southern Blot.

- Recuento de células de Sézary circulantes al menos de 1.000 células/microL o uno de los dos siguientes criterios:

- Aumento de CD4+ o CD3+ con una razón CD4/CD8 $>10$.

- Aumento de CD4 + con fenotipo anormal (razón CD4+CD7 $\geq 40 \%$ o a razón $\mathrm{CD} 4+\mathrm{CD} 26 \geq 30 \%)$.

Desde el año 2007, el SS ha sido definido como en estadío T4 B2 y compromiso hematológico, utilizando el mismo sistema de estadiaje que la MF (Tabla 2).

\section{Tabla 2. ISCL/EORTC Revisión de clasificación TNM de micosis fungoide y síndrome de Sézary}

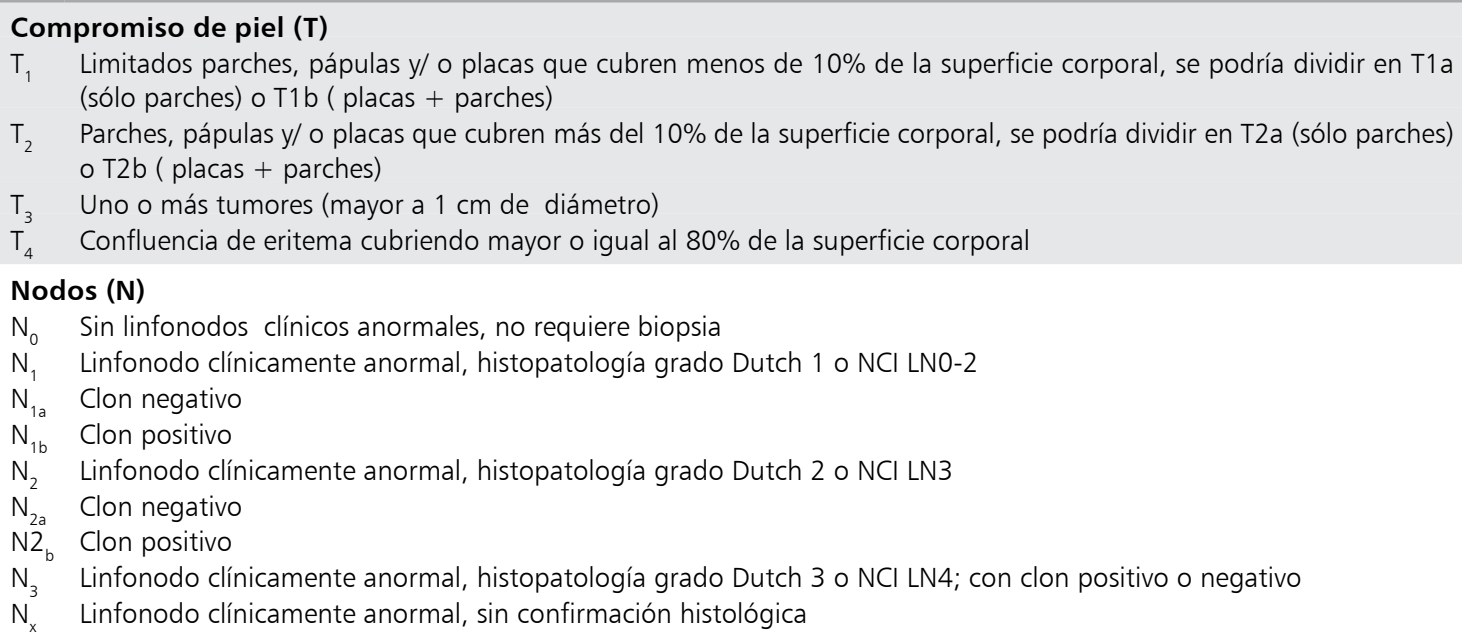

$\mathrm{B}_{0} \quad$ Sin compromiso de sangre significativo; menor o igual al 5\% de células de Sézary, menor 250 microL de células de Sésary, CD4+ CD26- o CD4+ CD6-

$\mathrm{B}_{0 \mathrm{a}} \quad$ Clon negativo

$\mathrm{B}_{\mathrm{ob}} \quad$ Clon positivo

$\mathrm{B}_{1} \quad$ Carga tumoral baja de sangre, que no cumple el criterio BO ० B2

$B_{1 a} \quad$ Clon negativo

$\mathrm{B}_{1 \mathrm{~b}} \quad$ Clon positivo

$\mathrm{B}_{2} \quad$ Carga tumoral alta en sangre, clon positivo; con más de uno de lo siguientes: Células Sézary mayor o igual a 1.000; CD4/CD8 mayor o igual a 10; CD4+ CD7- mayor o igual al 40\% o CD4+CD26 mayor o igual al 30\%

Tabla reacondicionada y fue originalmente publicada en Blood. Olsen E, Vonderheid E, Pimpinelli N, Willemze R, Kim Y, Knobler $R$, et al. Revisions to the staging and classification of mycosis fungoides and Sézary syndrome: a proposal of the International Society for Cutaneous Lymphomas (ISCL) and the cutaneous lymphoma task force of the European Organization of Research and Treatment of Cancer (EORTC). Blood 2007; 110 (6): 1713-22. 
Tabla 3. Sistema de estadificación clínica para la mucosis fungoide

\begin{tabular}{|cllll|}
\hline Estadio clínico & \multicolumn{4}{ll}{ Clasificación TNMB } \\
IA & $\mathrm{T}_{1}$ & $\mathrm{~N}_{0}$ & $\mathrm{M}_{0}$ & $\mathrm{~B}_{0} \circ \mathrm{B}_{1}$ \\
IB & $\mathrm{T}_{2}$ & $\mathrm{~N}_{0}$ & $\mathrm{M}_{0}$ & $\mathrm{~B}_{0} \circ \mathrm{B}_{1}$ \\
IIA & $\mathrm{T}_{1} \circ \mathrm{T}_{2}$ & $\mathrm{~N}_{1} \circ \mathrm{N}_{2}$ & $\mathrm{M}_{0}$ & $\mathrm{~B}_{0} \circ \mathrm{B}_{1}$ \\
IIB & $\mathrm{T}_{3}$ & $\mathrm{~N}_{1}$ a $\mathrm{N}_{2}$ & $\mathrm{M}_{0}$ & $\mathrm{~B}_{0} \circ \mathrm{B}_{1}$ \\
IIIB & $\mathrm{T}_{4}$ & $\mathrm{~N}_{1}$ a $\mathrm{N}_{2}$ & $\mathrm{M}_{0}$ & $\mathrm{~B}_{0}$ \\
IVA1 & $\mathrm{T}_{4}$ & $\mathrm{~N}_{1}$ a $\mathrm{N}_{2}$ & $\mathrm{M}_{0}$ & $\mathrm{~B}_{1}$ \\
IVA2 & $\mathrm{T}_{1}$ a $\mathrm{T}_{4}$ & $\mathrm{~N}_{1}$ a $\mathrm{N}_{2}$ & $\mathrm{M}_{0}$ & $\mathrm{~B}_{2}$ \\
IVB & $\mathrm{T}_{1}$ a $\mathrm{T}_{4}$ & $\mathrm{~N}_{3}$ & $\mathrm{M}_{0}$ & $\mathrm{~B}_{0}$ a $\mathrm{B}_{2}$ \\
\hline $\mathrm{T}_{1}$ a $\mathrm{T}_{4}$ & $\mathrm{~N}_{0}$ a $\mathrm{N}_{3}$ & $\mathrm{M}_{1}$ & $\mathrm{~B}_{0}$ a $\mathrm{B}_{2}$ \\
\hline
\end{tabular}

Tabla reacondicionada y fue originalmente publicada en Blood. Olsen E, Vonderheid E, Pimpinelli N, Willemze R, Kim $Y$, Knobler $R$, et al. Revisions to the staging and classification of mycosis fungoides and Sezary syndrome: a proposal of the International Society for Cutaneous Lymphomas (ISCL) and the cutaneous lymphoma task force of the European Organization of Research and Treatment of Cancer (EORTC). Blood 2007;110 (6): 1713-22.

Los pacientes con SS se consideran en estadío IVA1, IVA2 o IVB, dependiendo de la presencia de compromiso nodal o visceral (Tabla 3 ).

Los pacientes con SS generalmente presentan intenso prurito, eritrodermia y adenopatías generalizadas. El prurito intratable afecta de manera importante la calidad de vida de los pacientes ${ }^{2}$.

En la mayoría de los casos de SS no se puede determinar la causa, pero algunos casos han sido asociados a virus linfotrópicos tipo 1 y 2 (HTLAV1/2) ${ }^{3}$.

La incidencia exacta del SS no es conocida. En los Estados Unidos de Norteamérica entre 2001 y 2005 se estimó una incidencia de 0,8 casos por millón de personas por año, con un rango hombre/ mujer de 2,114. En general es una enfermedad del adulto mayor, ya que existen escasos reportes en menores de 30 años ${ }^{5}$.

Generalmente presentan un mal pronóstico, con un promedio de sobrevida de 32 meses al diagnóstico ${ }^{6}$.

No existe una terapia estándar inicial para el tratamiento de los pacientes con SS. El tratamiento del SS y de la MF eritrodérmica es fundamentalmente dirigida a mejorar la sintomatología, puesto que no existe terapia curativa. Consecuentemente, las terapias debieran ser lo menos tóxicas posibles y darse prioridad a aquellas que potencien el sistema inmune antes que la quimioterapia. Además, deben tomarse en cuenta las comorbilidades del paciente, los costos y el acceso al tratamiento.

Debido al compromiso leucémico en el SS, suelen utilizarse tratamientos sistémicos. Estos pueden ser utilizados como monoterapia, asociados a terapias dirigidas a la piel o en conjunto a otros tratamientos sistémicos. Los tratamientos deben ser guiados según el estadío de la enfermedad ${ }^{3}$.

Las siguientes recomendaciones fueron seleccionadas del Cutaneous Lymphoma Consortium, National Comprehensive Cancer Network (NCCN $)^{7}$, British Association of Dermatologists/ United Kingdom Cutaneous Lymphoma Group ${ }^{8}$, European Organization for Research and Treatment of Cancer (EORTC) ${ }^{9}$, European Society of Medical Oncology (ESMO), ESMO Guidelines Working Group. Primary cutaneous lymphomas ${ }^{10}$, Japanese Dermatologic Association (JDA)/Japanese Skin Cancer Society $(\text { JSCS })^{11}$ (Tabla 4).

\section{Estadio IVA1 e IVA2}

El tratamiento inicial para estos estadíos, consiste en terapia sistémica con o sin terapia dirigida a la piel (corticoide tópico, quimioterapia tópica como mostaza nitrogenada, o carmustina, radioterapia local, retinoides tópicos, fototerapia e imiquimod) ${ }^{7}$.

Se prefieren terapias que preserven el sistema inmune (Terapias A: Tabla 5). Los agentes del grupo A pueden también utilizarse combinados entre ellos, como el uso de FEC asociado a agentes moduladores de respuestas biológicos (interferón, retinoides) ${ }^{12,13}$.

\section{Estadio IVB}

Los pacientes con estadio IVB generalmente son manejados con inhibidores de la histona deacetilasa o mono quimioterapia (Terapias grupo B: Tabla 5).

Las terapias inmunoestimuladoras (fotoféresis, interferón y retinoides) y las terapias dirigidas a la piel pueden ser utilizadas de adyuvantes luego de completar los regímenes de quimioterapia.

\section{Enfermedad recurrente o refractaria}

Para estos pacientes no existe un tratamiento estándar. En general se prefiere el uso de terapias conservadoras de la función del sistema inmune antes que la quimioterapia tradicional. 
Tabla 4. Organización Europea para la investigación y tratamiento de cáncer, consenso recomendación para la micosis fungoide/síndrome Sésary

\begin{tabular}{|c|c|}
\hline Tratamientos recomendados & Nivel de evidencia \\
\hline \multicolumn{2}{|c|}{ Recomendación para la primera línea de tratamiento de la MF (etapa IIB) } \\
\hline PUVA $+I N F \alpha$ & B $1 b$ \\
\hline Electrom Bean e irradiación X superficial & C4 \\
\hline Retinoides + INF $\alpha$ & B $1 b$ \\
\hline PUVA + retinoides & D5 \\
\hline \multicolumn{2}{|c|}{ Recomendación para la primera línea de tratamiento de la MF (etapa III) } \\
\hline PUVA $+I N F \alpha$ & B $1 b$ \\
\hline INF $\alpha$ & B $2 b$ \\
\hline MTX & C4 \\
\hline Electrom Bean e irradiación X superficial & C4 \\
\hline Carbustina o mostaza nitrogenada & $\mathrm{C} 4$ \\
\hline FEC & C4 \\
\hline PUVA + retinoides & D5 \\
\hline \multicolumn{2}{|c|}{ Recomendación para la primera línea de tratamiento del SS } \\
\hline FEC & $\mathrm{C} 2 \mathrm{~b}$ \\
\hline INF $\alpha$ & $\mathrm{C} 2 \mathrm{~b}$ \\
\hline Denileucina diftitox & B $1 b$ \\
\hline Clorambucilo + prednisona & C4 \\
\hline
\end{tabular}

INF: interferón/MTX: Metrotexato/FEC: Fotoféresis estracorpórea. Tabla reacondicionada y publicada originalmente en Trautinger F, Knobler R, Willemze R, Peris K, Stadler R, Laroche L, et al. EORTC consensus recommendations for the treatment of mycosis fungoides/Sézary syndrome. Eur J Cancer Oxf Engl 1990 2006; 42 (8): 1014-30.

\section{Fotoféresis extracorpórea (FEC)}

Es una de las terapias iniciales sistémicas de elección en pacientes con linfomas de células $\mathrm{T}$ en estadio IVA1, IVA2 o SS.

La FEC es un método para irradiar el torrente sanguíneo directamente con PUVA (psoraleno/8-methoxsalen $(8 \mathrm{MOP})+$ fotoquimioterapia con luz ultravioleta $\mathrm{A}$ ), utilizando un proceso de leucoféresis extracorpórea que separa las células mononucleares circulantes, las mezcla con el 8 MOP, las irradia con UVA y luego son devueltas a la circulación del paciente ${ }^{14}$.

Los psoralenos son furocumarinas que absorben el máximo de luz UV en el rango de la UVA. Los psoralenos, incluyendo el 8MOP, se intercalan entre los pares de bases de ADN. Absorben fotones de radiación UVA generando monoaductos y enlaces que causan daño del ADN y muerte celular $^{14}$. La FEC combina una leucoféresis con un tratamiento ex vivo con 8 MOP fotoactivado por UVA.

La fotoféresis induce apoptosis de los leucocitos al producir daño al ADN. Al reintroducirlos a la circulación sanguínea, son fagocitados por cé- lulas presentadoras de antígenos que producen activación monocitaria, estimulación de citoquinas inmunomoduladoras o supresoras y estimulación de respuestas inmunes mediadas por la inmunidad celular ${ }^{14,15}$. La reinfusión se asocia a reducción del rango CD4/CD8 lo que sugiere una respuesta de inmunización tipo vacuna ${ }^{16}$.

Esta técnica está aprobada por la Food and Drugs Administration (FDA) de los Estados Unidos de Norteamérica desde el año 1988 para el tratamiento del SS. A partir del año 2003, las guías del Reino Unido lo recomendaron para aquellos pacientes con linfomas de células $\mathrm{T}$ avanzados, sobre todo luego de que terapias dirigidas a la piel fracasaran ${ }^{16}$.

La FEC estaría hoy fuertemente recomendada para pacientes con eritrodermia estadio III o IV a, asociado a alguno de los siguientes: enfermedad clonal circulante (PCR o Southern Blot), evidencia de células circulantes de Sézary $(>10 \%$ de linfocitos); CD4/CD8 > $10^{17}$.

Los principios básicos de la FEC se han mantenido sin cambios, pero los instrumentos se han ido desarrollando. Desde el año 1999, el método de Uvadex ha remplazado el uso de $8 \mathrm{MOP}$ con 
Tabla 5. Resumen de terapias para SS

\begin{tabular}{|c|c|c|c|}
\hline Agente & Comentario & Terapia combinada & Estado en FDA \\
\hline \multicolumn{4}{|c|}{ Tratamiento Primario: Terapias A } \\
\hline $\begin{array}{l}\text { Electroforesis extra- } \\
\text { corpórea (FEC) }\end{array}$ & $\begin{array}{l}\text { Sólo disponible en centros } \\
\text { especializados, no causa in- } \\
\text { munosupresión generalizada }\end{array}$ & $\begin{array}{l}\text { Puede ser combiando con } \\
\text { otras tratamientos sistémi- } \\
\text { cos (retinoides, dosis bajas } \\
\text { de MTX, inhibidores de la } \\
\text { HDAC, INF) o tratamientos } \\
\text { directos a la piel (tópicos, } \\
\text { fototerapia, electron beam) }\end{array}$ & Aprobado \\
\hline $\begin{array}{l}\text { Retinoides (bexaroteno, } \\
\text { acitretina, isotretinoina, } \\
\text { todos los derivados del } \\
\text { ácido retinoico) }\end{array}$ & $\begin{array}{l}\text { Teratógenos, muchos efectos } \\
\text { adversos, como hiperlipemia } \\
\text { e hipotiroidismo central } \\
\text { (bexaroteno) }\end{array}$ & $\begin{array}{l}\text { Puede ser combinado con } \\
\text { otros tratamientos sistémicos } \\
\text { (FEC, INF) o tratamientos } \\
\text { directos a la piel (PUVA, } \\
\text { electron beam) }\end{array}$ & $\begin{array}{l}\text { Bexaroteno, aprobado para } \\
\text { enfermedad refractaria }\end{array}$ \\
\hline Interferón (INF $\alpha$ e INF $\gamma$ ) & $\begin{array}{l}\text { Usar con precaución en } \\
\text { pacientes con condiciones } \\
\text { autoinmunes (como AR) o } \\
\text { trasplantados de órganos } \\
\text { sólidos) }\end{array}$ & $\begin{array}{l}\text { Puede ser combinado con } \\
\text { otros tratamientos sistémicos } \\
\text { (FEC, Bexaroteno) o trata- } \\
\text { mientos directos a la piel } \\
\text { (PUVA, electron beam) }\end{array}$ & $\begin{array}{l}\text { INF } \alpha \text {, Aprobado para lin- } \\
\text { fomas no Hodgkin (incluye } \\
\text { MF y SS) }\end{array}$ \\
\hline $\begin{array}{l}\text { Inhibidores de la histona } \\
\text { deacetilasa (HDAC) } \\
\text { (vorinostat, romidepsin) }\end{array}$ & $\begin{array}{l}\text { Fatiga, náuseas, diarrea, } \\
\text { trombocitopenia, efectos } \\
\text { inespecíficos del electrocar- } \\
\text { diograma }\end{array}$ & $\begin{array}{l}\text { Puede ser combinado con } \\
\text { otros tratamientos sistémicos } \\
\text { (FEC, INF) o tratamientos } \\
\text { directos a la piel (electron } \\
\text { beam) }\end{array}$ & $\begin{array}{l}\text { Romidepsin: aprobado para } \\
\text { el tratamientode la enferme- } \\
\text { dad progresiva, persistente o } \\
\text { recurrente o posterior al uso } \\
\text { de un tratamiento sistémico. } \\
\text { Vonirostat aprobado posterior } \\
\text { a } 2 \text { terapias sistémicas }\end{array}$ \\
\hline Dosis bajas de MTX & $\begin{array}{l}\text { Teratogénico, leve a gene- } \\
\text { ralizada inmunosupresión, } \\
\text { toxicidad hepática }\end{array}$ & $\begin{array}{l}\text { Puede ser combinado con } \\
\text { otros tratamientos sistémicos } \\
\text { (FEC, INF, IHDA) o tratamien- } \\
\text { tos directos a la piel (PUVA, } \\
\text { electron beam) }\end{array}$ & Aprobado \\
\hline \multicolumn{4}{|c|}{$\begin{array}{l}\text { Tratamiento secundario: Terapias B: Puede ser usada después de una inadecuada respuesta, } \\
\text { enfermedad refractaria o progresión a pesar del tratamiento primario }\end{array}$} \\
\hline $\begin{array}{l}\text { Doxorrubicina liposomal } \\
\text { pegilada }\end{array}$ & Mielosupresión & & Fuera de etiqueta \\
\hline Gemcitabina & Mielosupresión & & Fuera de etiqueta \\
\hline Alemtuzumab & $\begin{array}{l}\text { No disponible } \\
\text { comercialmente }\end{array}$ & & Fuera de etiqueta \\
\hline Clorambucil & Mielosupresión & & Fuera de etiqueta \\
\hline Fludarabina & Mielosupresión & $\begin{array}{l}\text { Puede ser combinado con } \\
\text { ciclofosfamida }\end{array}$ & Fuera de etiqueta \\
\hline Cladribina & Mielosupresión & & Fuera de etiqueta \\
\hline Pentostatina & Mielosupresión & & Fuera de etiqueta \\
\hline Dosis intermedia de MTX & Mielosupresión & & Aprobado \\
\hline Dosis bajas de Pralatrexato & Estomatitis, toxicidad de piel & & $\begin{array}{l}\text { Aprobado para enfermedad } \\
\text { refractaria }\end{array}$ \\
\hline
\end{tabular}

Tabla reacondicionada y publicada originalmente en Olsen EA, Rook AH, Zic J, Kim Y, Porcu P, Querfeld C, et al. Sézary syndrome: immunopathogenesis, literature review of therapeutic options, and recommendations for therapy by the United States Cutaneous Lymphoma Consortium (USCLC). J Am Acad Dermatol 2011; 64 (2): 352-404. 
una solución de metoxipsoraleno antes de la irradiación extracorpórea con UVA (1-2 $\left.\mathrm{Jcm}^{2}\right)$, reduciendo así la toxicidad por $8 \mathrm{MOP}$.

Estudios iniciales con el uso de FEC dos días seguidos cada 4 semanas, mostraron $83 \%$ de mejoría como monoterapia en pacientes eritrodérmicos (mejoría definida como $<25 \%$ compromiso de superficie corporal) con $21 \%$ de pacientes que consiguieron la remisión completa ${ }^{16}$. Sin embargo, son pocos los estudios que han evaluado la efectividad de la FEC como monoterapia. La respuesta, definida como mejoría $>50 \%$ del compromiso cutáneo, es aproximadamente de $25 \%{ }^{18-22}$. El promedio de respuesta completa en este grupo fue cercana a $10 \%$.

Estudios siguientes demostraron respuestas que varían entre 31 y $86 \%$ en 5 a 6 meses, dependiendo del paciente seleccionado, la frecuencia de tratamiento y el uso de terapias coadyuvantes ${ }^{23,24}$.

$\mathrm{Al}$ combinar la técnica con agentes biológicos modificadores de respuestas, la respuesta parece ser más rápida y con mayores tasas que datos históricos previos ${ }^{25-27}$.

El seguimiento a largo plazo de una serie de pacientes mostró que el promedio de sobrevida fue de 60 meses $^{28}$.

Los protocolos varían de centro en centro, por lo general la FEC se administra por dos días consecutivos cada 2 o cada 4 semanas $^{29}$. Se debe realizar en centros especializados. El tiempo total de tratamiento es de 1,5 a $4 \mathrm{~h}$ y debe durar un mínimo de 6 meses.

La primera evaluación se realiza a los 3 meses. Aquí se evalúa la continuación de la terapia a los 6 meses y se considera la opción de adicionar una terapia coadyuvante (interferón, retinoides o terapia dirigida a la piel). Si existe respuesta, el tratamiento se mantiene en la misma frecuencia hasta la máxima respuesta. En general, para los LCCT, se reporta un promedio de 10 meses $^{24}$. Una vez obtenida esta máxima respuesta, se puede ir disminuyendo la frecuencia a un ciclo de mantención (dos tratamientos consecutivos) cada 6 o 12 meses.

Los efectos adversos son infrecuentes. Dentro de ellos se encuentran hipotensión leve, pirexia a las 6 a 8 h de reinfusión de los linfocitos, fatiga, cefaleas, aumento transitorio del prurito y necesidad de un acceso venoso.

Los predictores de buena respuesta para FEC como monoterapia incluyen: enfermedad T4 (eritrodermia), recuento de leucocitos < 20.000/ microL, recuento de células de Sézary entre $10 \mathrm{y}$ $20 \%$, menos de dos años desde el inicio del diagnóstico, sin grandes adenopatías, sin enfermedad visceral, no haber utilizado quimioterapia previa, sin elevación de células NK o de CD $8^{29-31}$.

Clínicamente, el predictor más importante de respuesta a largo plazo es una respuesta precoz (definida como reducción $>50 \%$ ) de lesiones cutáneas a los 6 meses de inicio de tratamiento ${ }^{29}$.

Por lo general, se requiere de un período de tratamiento de 6 meses para FEC antes de identificar una enfermedad refractaria.

\section{Conclusión}

El SS es una forma agresiva del LCCT que se caracteriza por presentar eritrodermia, linfadenopatía y linfocitos atípicos circulantes. El tratamiento de este síndrome depende del estadio clínico de la enfermedad, la sintomatología del paciente y la disponibilidad de ellos. La FEC ofrece una buena alternativa para tratamiento al ser una terapia inmunomoduladora que se ha mostrado eficaz para su tratamiento y se caracteriza por su escasa toxicidad comparada con la mayoría de los inmunosupresores.

El procedimiento tiene tres fases: leucoféresis, fotoactivación y reinfusión posterior. Los linfocitos tratados detienen su ciclo celular y sufren apoptosis, luego son fagocitados por células presentadoras de antígeno, con producción posterior de células supresoras tumorales específicas.

Nuestro centro cuenta con la única máquina de FEC en Chile (marca Therakos ${ }^{\circledR}$ ) y es la cuarta existente en Latinoamérica. Además del difícil acceso, los costos económicos son aún elevados. Sin embargo, el ingreso precoz de los pacientes a esta terapia se asocia a buen resultado terapéutico, como se ha demostrado en nuestro paciente y esta publicado en la literatura internacional.

Presentamos este caso para ejemplificar la buena experiencia obtenida en este paciente con FEC en nuestro centro.

\section{Referencias}

1. Olsen E, Vonderheid E, Pimpinelli N, Willemze R, Kim $\mathrm{Y}$, Knobler R, et al. Revisions to the staging and clas- 
sification of mycosis fungoides and Sézary syndrome: a proposal of the International Society for Cutaneous Lymphomas (ISCL) and the cutaneous lymphoma task force of the European Organization of Research and Treatment of Cancer (EORTC). Blood 2007; 110 (6): 1713-22.

2. Demierre MF, Gan S, Jones J, Miller DR. Significant impact of cutaneous T-cell lymphoma on patients' quality of life: results of a 2005 National Cutaneous Lymphoma Foundation Survey. Cancer 2006; 107 (10): 2504-11.

3. Olsen EA, Rook AH, Zic J, Kim Y, Porcu P, Querfeld C, et al. Sézary syndrome: immunopathogenesis, literature review of therapeutic options, and recommendations for therapy by the United States Cutaneous Lymphoma Consortium (USCLC). J Am Acad Dermatol 2011; 64 (2): 352-404.

4. Bradford PT, Devesa SS, Anderson WF, Toro JR. Cutaneous lymphoma incidence patterns in the United States: a population-based study of 3884 cases. Blood 2009; 113 (21): 5064-73.

5. Criscione VD, Weinstock MA. Incidence of cutaneous T-cell lymphoma in the United States, 1973-2002. Arch Dermatol 2007; 143 (7): 854-9.

6. Willemze R, Kerl H, Sterry W, Berti E, Cerroni L, Chimenti $S$, et al. EORTC classification for primary cutaneous lymphomas: a proposal from the cutaneous lymphoma study group of the European Organization for Research and Treatment of Cancer. Blood 1997; 90: 354-71.

7. Zelenetz AD, Abramson JS, Advani RH, Andreadis CB, Byrd JC, Czuczman MS, et al. Non-Hodgkin's Lymphomas. J Natl Compr Canc Netw 2010; 8 (3): 288-334.

8. Whittaker SJ, Marsden JR, Spittle M, Russell Jones R, British Association of Dermatologists, U.K. Cutaneous Lymphoma Group. Joint British Association of Dermatologists and U.K. Cutaneous Lymphoma Group guidelines for the management of primary cutaneous T-cell lymphomas. Br J Dermatol 2003; 149 (6): 1095-107.

9. Trautinger F, Knobler R, Willemze R, Peris K, Stadler R, Laroche L, et al. EORTC consensus recommendations for the treatment of mycosis fungoides/Sézary syndrome. Eur J Cancer Oxf Engl 1990 2006; 42 (8): 1014-30.

10. Willemze R, Dreyling M, ESMO Guidelines Working Group. Primary cutaneous lymphomas: ESMO Clinical Practice Guidelines for diagnosis, treatment and follow-up. Ann Oncol Off J Eur Soc Med Oncol ESMO. 2010; 21 Suppl 5: v177-80.

11. Sugaya M, Hamada T, Kawai K, Yonekura K, Ohtsuka $\mathrm{M}$, Shimauchi T, et al. Guidelines for the management of cutaneous lymphomas (2011): a consensus statement by the Japanese Skin Cancer Society-Lymphoma Study Group. J Dermatol 2013; 40 (1): 2-14.

12. Raphael BA, Morrissey KA, Kim EJ, Vittorio CC, Rook AH. Psoralen plus ultraviolet A light may be associated with clearing of peripheral blood disease in advanced cutaneous T-cell lymphoma. J Am Acad Dermatol 2011; 65 (1): 212-4.

13. Klein RS, Dunlop JD, Samimi SS, Morrissey KA, Evans $\mathrm{KG}$, Gardner JM, et al. Improvement in peripheral blood disease burden in patients with Sézary syndrome and leukemic mycosis fungoides after total skin electron beam therapy. J Am Acad Dermatol 2013; 68 (6): 972-7.

14. Knobler R, Barr ML, Couriel DR, Ferrara JLM, French LE, Jaksch P, et al. Extracorporeal photopheresis: past, present, and future. J Am Acad Dermatol 2009; 61 (4): 652-65.

15. Bladon J, Taylor PC. Extracorporeal photopheresis induces apoptosis in the lymphocytes of cutaneous T-cell lymphoma and graft-versus-host disease patients. $\mathrm{Br} \mathrm{J}$ Haematol 1999; 107 (4): 707-11.

16. Edelson R, Berger C, Gasparro F, Jegasothy B, Heald $\mathrm{P}$, Wintroub B, et al. Treatment of cutaneous T-cell lymphoma by extracorporeal photochemotherapy. Preliminary results. N Engl J Med 1987; 316 (6): 297-303.

17. Scarisbrick JJ, Taylor P, Holtick U, Makar Y, Douglas K, Berlin G, et al. U.K. consensus statement on the use of extracorporeal photopheresis for treatment of cutaneous T-cell lymphoma and chronic graft-versus-host disease. Br J Dermatol 2008; 158 (4): 659-78.

18. Dippel E, Schrag H, Goerdt S, Orfanos CE. Extracorporeal photopheresis and interferon-alpha in advanced cutaneous T-cell lymphoma. Lancet 1997; 350 (9070): 32-3.

19. Vonderheid EC, Zhang Q, Lessin SR, Polansky M, Abrams JT, Bigler RD, et al. Use of serum soluble interleukin-2 receptor levels to monitor the progression of cutaneous T-cell lymphoma. J Am Acad Dermatol 1998; 38 (2 Pt 1): 207-20.

20. Bouwhuis SA, el-Azhary RA, Gibson LE, McEvoy MT, Pittelkow MR. Effect of insulin-dependent diabetes mellitus on response to extracorporeal photopheresis in patients with Sézary syndrome. J Am Acad Dermatol 2002; 47 (1): 63-7.

21. Stevens SR, Baron ED, Masten S, Cooper KD. Circulating CD4+CD7- lymphocyte burden and rapidity of response: predictors of outcome in the treatment of Sézary syndrome and erythrodermic mycosis fungoides with extracorporeal photopheresis. Arch Dermatol 2002; 138 (10): 1347-50.

22. de Misa RF, Harto A, Azana JM, et al. Photopheresis does not improve survival in Sezary syndrome patients 
with bone marrow involvement. J Am Acad Dermatol 2005; 53: 171-2.

23. McKenna KE, Whittaker S, Rhodes LE, Taylor P, Lloyd J, Ibbotson S, et al. Evidence-based practice of photopheresis 1987-2001: a report of a workshop of the British Photodermatology Group and the U.K. Skin Lymphoma Group. Br J Dermatol 2006; 154 (1): 7-20.

24. Duvic M, Chiao N, Talpur R. Extracorporeal photopheresis for the treatment of cutaneous T-cell lymphoma. J Cutan Med Surg 2003; 7: 3-7.

25. Suchin KR, Cucchiara AJ, Gottleib SL, Wolfe JT, DeNardo BJ, Macey WH, et al. Treatment of cutaneous T-cell lymphoma with combined immunomodulatory therapy: a 14-year experience at a single institution. Arch Dermatol 2002; 138 (8): 1054-60.

26. Richardson SK, Lin JH, Vittorio CC, Kim EJ, Yoon JS, Junkins-Hopkins J, et al. High clinical response rate with multimodality immunomodulatory therapy for Sézary syndrome. Clin Lymphoma Myeloma 2006; 7 (3): 226-32.

27. Raphael BA, Shin DB, Suchin KR, Morrissey KA, Vit- torio CC, Kim EJ, et al. High clinical response rate of Sezary syndrome to immunomodulatory therapies: prognostic markers of response. Arch Dermatol 2011; 147 (12): 1410-5.

28. Heald P, Rook A, Pérez M, Wintroub B, Knobler R, Jegasothy $\mathrm{B}$, et al. Treatment of erythrodermic cutaneous T-cell lymphoma with extracorporeal photochemotherapy. J Am Acad Dermatol 1992; 27 (3): 427-33.

29. Zic JA. Photopheresis in the treatment of cutaneous T-cell lymphoma: current status. Curr Opin Oncol 2012; 24 Suppl 1:S1-10.

30. McGirt LY, Thoburn C, Hess A, Vonderheid EC. Predictors of response to extracorporeal photopheresis in advanced mycosis fungoides and Sézary syndrome. Photodermatol Photoimmunol Photomed 2010; 26 (4): 182-91.

31. Evans AV, Wood BP, Scarisbrick JJ, Fraser-Andrews EA, Chinn S, Dean A, et al. Extracorporeal photopheresis in Sézary syndrome: hematologic parameters as predictors of response. Blood 2001; 98 (5): 1298-301. 